\title{
Generation of Plasma Flows by 2 kW-class Continuous Wave Laser Driven Wind Tunnel
}

\author{
Koji SHINMI, ${ }^{1}$ Makoto MATSUI, ${ }^{1}$ Kimiya KOMURASAKI, ${ }^{1}$ and Yoshihiro ARAKAWA ${ }^{2}$ \\ ${ }^{1}$ The University of Tokyo, 5-1-5 Kashiwanoha, Kashiwa, Tokyo 277-8562 \\ ${ }^{2}$ The University of Tokyo, 7-3-1 Hongo, Bunkyo-ku, Tokyo 113-8656
}

(Received February 1, 2008)

\begin{abstract}
A continuous wave laser driven wind tunnel has been developed to produce high enthalpy flows to simulate atmospheric reentry environment. As a result of preliminary operation tests, high enthalpy argon/oxygen flows were successfully generated with the input laser power of $800 \mathrm{~W}$, the plenum pressure of $950 \mathrm{kPa}$ and the plume diameter of $15 \mathrm{~mm}$. Next, plume characteristics were diagnosed by laser absorption spectroscopy. Consequently, the radial distributions of the specific enthalpy were measured. It was found that the specific enthalpy of flow was around $4 \mathrm{MJ} / \mathrm{kg}$ at the radial position $\mathrm{r}<3 \mathrm{~mm}$.
\end{abstract}

Key Words: CW laser, High enthalpy flow, Laser absorption spectroscopy

\section{Introduction}

In developing thermal protection systems (TPS) for entry/reentry vehicles, arc-heaters are widely used to simulate such high enthalpy flows because it is simple and rugged structure, long operational time and requires a little maintenance after several-hour operation ${ }^{1)}$. Recently, atomic oxygen is found to play important roles through the heat-flux enhancement by catalytic effect and the active-passive oxidation of TPS surfaces. Atomic oxygen flows are also required to simulate space environments at low earth orbit (LEO). Since spacecrafts have velocities of $\sim 7.8 \mathrm{~km}$ at LEO, atomic oxygen dissociated by ultraviolet ray from the sun collide with them with a translational energy of $\sim 4.5 \mathrm{eV}$, resulting in the severe degradation of their surface materials. However, erosion of their electrodes poses an important obstacle because polluted flows make it difficult to evaluate chemical reaction rates in front of TPS surfaces.

For the reasons above, inductively coupled plasma generators have garnered much attention ${ }^{2}$. Such generators have no electrode. They can produce an ideal test condition for TPS tests because they have no undesirable chemical reactions that result from erosion. Another advantage of such generators is that they can use even reactive gases such as carbon dioxide and oxygen because of their electrode-less heating.

Although Mars entry conditions can be simulated using these generators, their operation is possible at the total pressure lower than $0.1 \mathrm{MPa}^{3}$.

In our group, the laser sustained plasma (LSP) has been studied for "laser propulsion". The LSP was stably produced under the plenum pressure $600 \mathrm{kPa}^{4}$.

In this study, high enthalpy argon/oxygen flows were generated and their spatial distribution of specific enthalpy was measured.

\section{Laser Driven Wind Tunnel}

\section{1 Principle of Laser Driven Wind Tunnel}

Figure 1 shows the principle of laser driven wind tunnel. The laser beam supplied from the outside, focused through lens, initial plasma is generated using metallic sticks for ignition. Once plasma is generated, plasma begins to absorb the laser beam by the inverse bremsstrahlung absorption process. As a result, the ionizing proceeds, the electron density is increased in the avalanche, and plasma propagates in the direction of the laser upstream by heat conduction, witch called Laser Supported Combustion wave. Then LSP is maintained at the balance of laser energy absorption and the energy that lost in the surrounding low temperature gas and radiation.

The energy of the LSP is transmitted to the gas, then, heated gas expands and accelerates through the nozzle, resulting in high enthalpy flow generation.

\section{2 LSP generator}

Figure 2 shows the cross section of the LSP generator for the $2 \mathrm{~kW}$-class laser device. Basically, the generator is composed of a laser induction window, a plasma-sustaining chamber, and a convergent-divergent nozzle. A zinc selenide $(\mathrm{ZnSe})$ lens with anti-reflection coating was used as the laser induction window. It can transmit $10.6 \mu \mathrm{m}$ wavelength laser beam efficiently and withstand up to $1 \mathrm{MPa}$ inner pressure. The nozzle is made of cupper with $1 \mathrm{~mm}$ in throat diameter and with $20 \mathrm{~mm}$ diameter at the exit of the nozzle. Other generator segments are mainly made of stainless steel.

To ignite plasma, a steel rod is used as the source of electron emission. This rod was inserted into the focal point of the laser beam and was pulled back after ignition by air cylinder. Next, axially moved the focal lends, LSP was moved to the downstream so that plum may come to be the largest and to

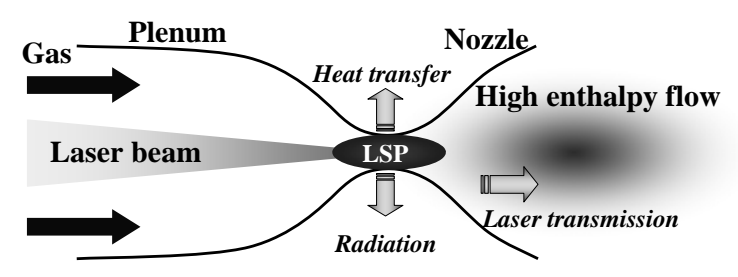

Fig.1 Conceptual figure of laser driven wind tunnel. 


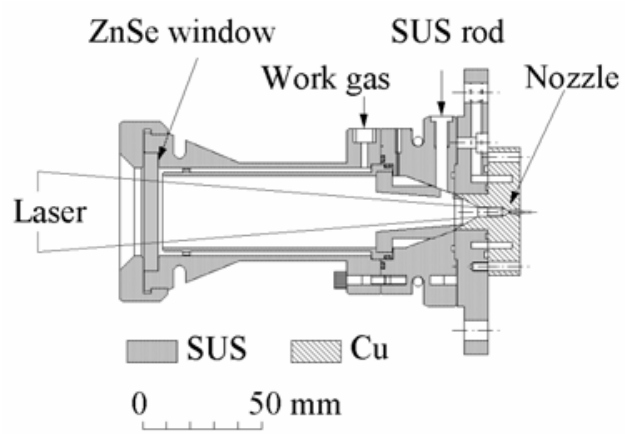

Fig. 2 Overview of the LSP generator.

stabilize. In these experiments, a $2 \mathrm{~kW} \quad \mathrm{CW}-\mathrm{CO}_{2}$ laser (Panasonic YB-L200B7T4), with $10.6 \mu \mathrm{m}$ wavelength variable power, was utilized. The focal length of lens is $250 \mathrm{~mm}$, and the incident beam diameter is $34 \mathrm{~mm}$.

LSP generator is connected with vacuum chamber, by using the rotary vacuum pump (The exhaust speed: $40 \mathrm{~m}^{3} / \mathrm{h}$ ) and mechanical booster pump (The exhaust speed: $500 \mathrm{~m}^{3} / \mathrm{h}$ ) backpressure can be kept under $200 \mathrm{~Pa}$.

\section{Laser Absorption Spectroscopy ${ }^{5) 6}$}

3.1 Principle of laser absorption spectroscopy

Laser absorption spectroscopy is applicable to optically thick plasma and does not require absolute calibration using a calibrated light source or a density reference cell. In addition, measurement system using a diode laser can be portable ${ }^{7}$.

The relationship between laser intensity $I(v)$ and absorption coefficient $k(v, x)$ is expressed by the Beer-Lambert law as ${ }^{8)}$

$$
\frac{\mathrm{d} I(v)}{\mathrm{d} x}=-k(v, x) I(v) \text {. }
$$

Here, $v$ is the laser frequency and $x$ is the coordinate in the laser pass direction.

In our experimental conditions, Doppler broadening is several gigahertzes, which is two orders of magnitude greater than all other broadenings, including natural, pressure and Stark broadenings. The absorption profile $k(v, x)$ is approximated as a Gaussian profile, expressed as

$$
k(v, x)=\frac{2 K(x)}{\Delta v_{\mathrm{D}}} \sqrt{\frac{\ln 2}{\pi}} \exp \left[-\ln 2\left\{\frac{2\left(v-v_{0}-\Delta v_{\text {shitt }}\right)}{\Delta v_{\mathrm{D}}}\right\}^{2}\right] .
$$

Here, $v_{0}$ is the center absorption frequency and $K(x)$ is the integrated absorption coefficient. $\Delta v_{\mathrm{D}}$ is the full width at half maximum of the profile and is related to the translational temperature $T$, expressed as

$$
\Delta v_{\mathrm{D}}=2 v_{0} \sqrt{\frac{2 k_{\mathrm{B}} T}{m c^{2}} \ln 2},
$$

where $m, c$ and $k_{\mathrm{B}}$ represent the mass of absorbers, velocity of light, and the Boltzmann constant, respectively. $\Delta v_{\text {shift }}$ is the shift of center absorption frequency by Doppler effect and expressed as

$$
\Delta v_{\text {shift }}=\frac{u v_{0}}{c} \sin \theta
$$

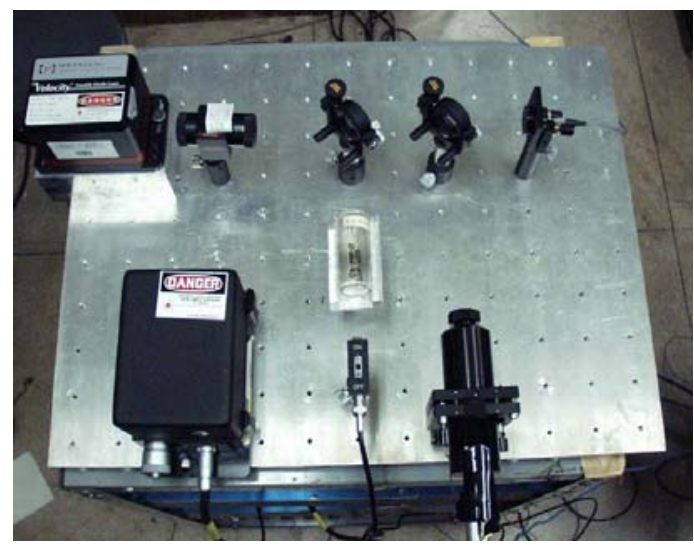

Fig. 3 Photo of the optical system.

Here, $u$ is the velocity of the absorber and $\theta$ is the incident angle of probe laser to the radial direction.

In this study, the target absorption line is ArI $772.38 \mathrm{~nm}$.

\subsection{Experimental setup}

A tunable diode-laser with an external cavity (Velocity Model 6300; New Forcus, Inc.) was used as the laser oscillator. Its line width was less than $500 \mathrm{kHz}$. The laser frequency was scanned over the absorption line shape $k(v)$. The modulation frequency and width were $1 \mathrm{~Hz}$ and $30 \mathrm{GHz}$, respectively. The laser intensity, which was normalized by saturation intensity, was reduced less than 0.02 by neutral density filters; it was sufficiently small to avoid the influence of absorption saturation. An optical isolator was used to prevent the reflected laser beam from returning into the external cavity. An etalon was used to calibrate relative frequency. Its free spectral range was $0.75 \mathrm{GHz}$. A glow discharge plasma with an input power, discharge voltage, and ambient pressure of $1.5 \mathrm{~W}, 300 \mathrm{~V}$, and $79 \mathrm{~Pa}$, respectively, was used as a plasma source with $u=0$. The laser frequency was calibrated by the center absorption frequency in the glow plasma. A photo of the optical system is shown in Fig. 3.

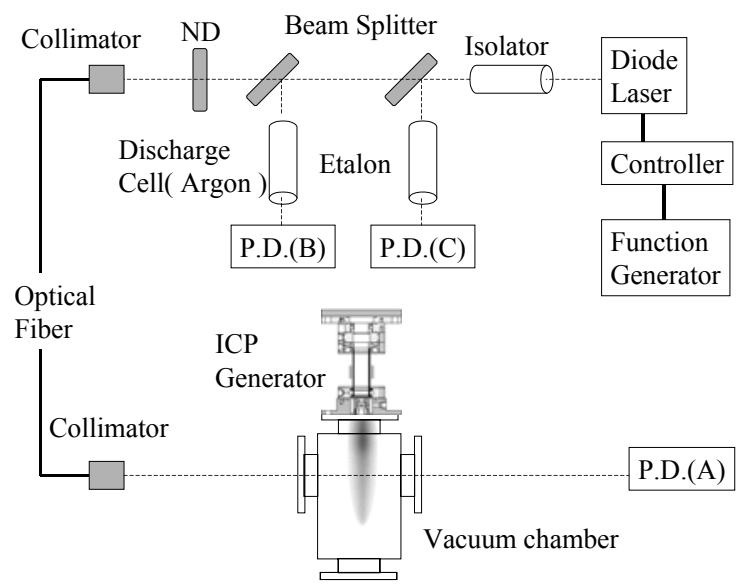

Fig.4 Measurement system. 


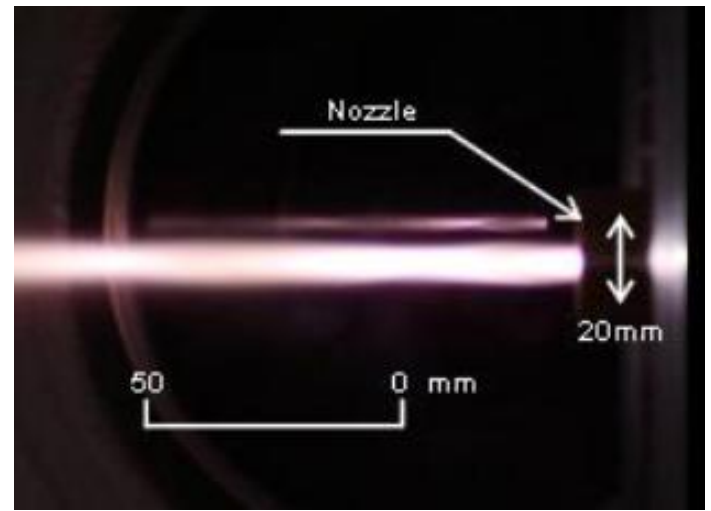

Fig. 5 Photograph of plume. $\left(800 \mathrm{~W}, \mathrm{Ar}: 10 \mathrm{slm}, \mathrm{O}_{2}: 0.1\right.$ slm)

The probe beam was guided to the chamber window through a singlemode optical fibre. The incident angle of the probe beam was 5 degree and its diameter was $1 \mathrm{~mm}$ at the plume centre. Transmitted laser intensity $I$ was measured using a photo detector (DET110/M; Thorlabs Inc.) Signals were recorded using a digital oscilloscope (DL1540; YOKOGAWA Co.) with 16-bit resolution at the sampling rate of $1 \mathrm{kHz}$. Figure 4 shows a schematic of the measurement system.

\section{Result and Discussion}

\section{1 fundamental wind tunnel performance}

Figure 5 shows typical argon/oxygen flow with the input laser power of $800 \mathrm{~W}$. First, argon LSP is generated, then, oxygen is mixed. Input power of the laser is fixed, and mass flow of argon is varied to change the plenum pressure. At these conditions, high plenum pressure limitation and flux density is measured. Figure 6 shows pressure dependency of the flux density. The maximum plenum pressure is $0.95 \mathrm{MPa}$ and the maximum flux density is $2.2 \times 10^{21} / \mathrm{cm}^{2}$ s with 0.31 $\mathrm{MPa}$ plenum pressure.

\section{2 Flow Diagnostics}

4. 2. 1 Temperature and velocity measurements

An operation condition is listed in Table 1. Typical signals that were recorded with frequency modulation are shown in Fig. 7 along with an etalon signal. Figure shows that the

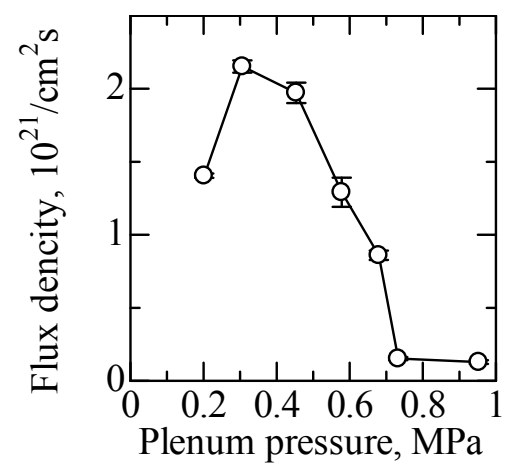

Fig. 6 Pressure dependency of the flux density.
Table 1 Operation condition

\begin{tabular}{ll}
\hline \hline Properties & Value \\
\hline Laser power, $\mathrm{W}$ & 800 \\
Argon, slm & $10,20,30,40,50$ \\
Oxygen, slm & 0.1 \\
Plenum pressure, $\mathrm{kPa}$ & $201-953$ \\
Ambient pressure, $\mathrm{Pa}$ & $25-122$ \\
\hline \hline
\end{tabular}

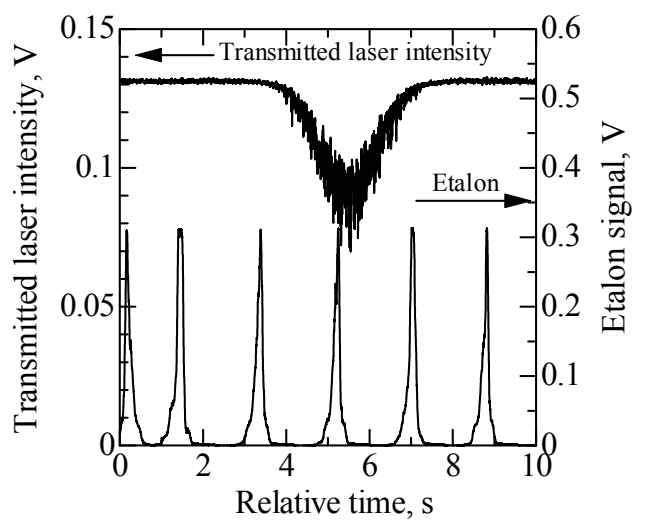

Fig. 7 Typical transmitted laser and etalon signals. (ArI $772.38 \mathrm{~nm})$

transmitted laser intensity oscillates $270 \mathrm{~Hz}$. Previous study in this laboratory shows that, $\mathrm{CO}_{2}$ laser intensity oscillates $50 \mathrm{~Hz}$ and $2 \%$, consequently, the position and emission intensity of $\mathrm{LSP}^{9)}$. By this reason, the oscillation of the absorbance signals is caused by the oscillation of $\mathrm{CO}_{2}$ laser intensity. In this study, maximum absorption rate of each oscillation is fitted, then, we obtained maximum absorption profile, as shown in Fig. 8.

Figure 9 shows radial distributions of translational temperature. This figure shows that, there are no remarkable difference among three mass flow conditions, translational temperature was almost constant around $300 \mathrm{~K}$ at the radial position $r<3 \mathrm{~mm}$, and it decreases gradually at the radial position $r>3 \mathrm{~mm}$.

Figure 10 shows radial distributions of velocity. As the result of translational temperature, there are no remarkable difference between three mass flow conditions, velocity was almost constant about $2500 \mathrm{~m} / \mathrm{s}$ at the radial position $r<3 \mathrm{~mm}$,

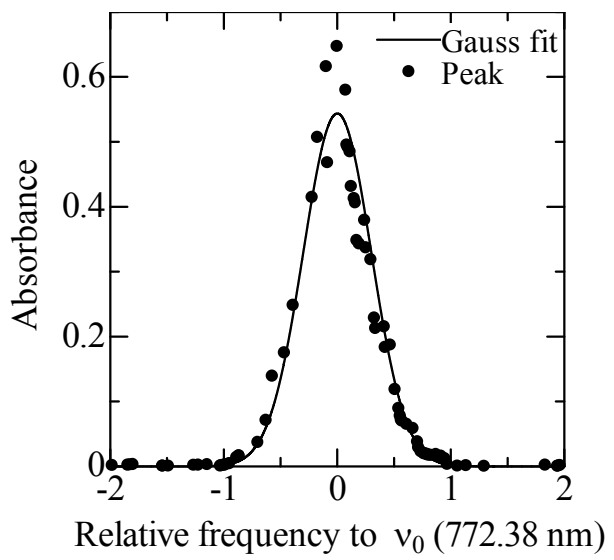

Fig. 8 Maximum absorbance and gauss fit. 


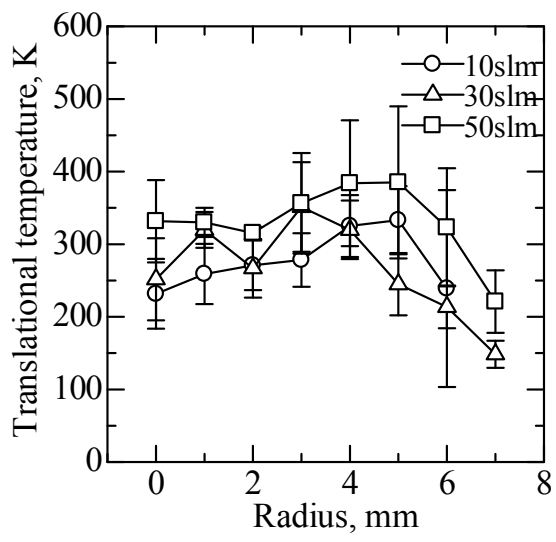

Fig. 9 Radial distributions of translational temperature.

and it decreases gradually at the radial position $r>3 \mathrm{~mm}$.

4. 2. 2 Radial distribution of the specific enthalpy

The specific enthalpy was estimated as follows. Assuming an isentropic expansion and chemically frozen flow through the nozzle, the total specific enthalpy $h_{0}$ is conserved expressed as

$$
h_{0}=\int_{0}^{T_{0}} C_{p} d T+h_{\text {chemica }}=\int_{0}^{T_{\text {exit }}} C_{p} \mathrm{~d} T+h_{\text {chemical }}+\frac{1}{2} u^{2}
$$

Here, $C \mathrm{p}$ and $T_{0}$ and $h_{\text {chem }}$ are the specific heat at constant pressure, the total temperature and the chemical potential, respectively. $h_{\text {chem }}$ is constant under the chemically frozen flow assumption.

Since the total pressure $p_{0}$ measured in the plenum chamber by a silicon-diaphragm pressure sensor was higher than 300 $\mathrm{kPa}$, the chemical composition in the plenum chamber of the flow was calculated assuming thermo-chemical equilibrium. In the calculation, six chemical species $\mathrm{Ar}, \mathrm{O}_{2}, \mathrm{O}, \mathrm{Ar}^{+}, \mathrm{O}^{+}$and $\mathrm{e}^{-}$, and three chemical reactions $\mathrm{Ar} \leftrightarrow \mathrm{Ar}^{+}+\mathrm{e}^{-}, \mathrm{O}_{2} \leftrightarrow 2 \mathrm{O}, \mathrm{O} \leftrightarrow \mathrm{O}^{+}+\mathrm{e}^{-}$, were considered. Their equilibrium constants were obtained from references 10 and 11 . The volumetric gas mixture ratio argon and oxygen and $p_{0}$ were set identical to the operation condition. $C p$ was computed as the sum of the contributions of

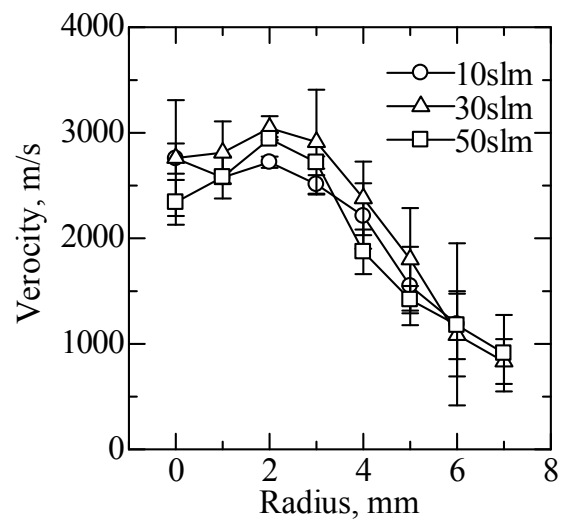

Fig. 10 Radial distributions of velocity.

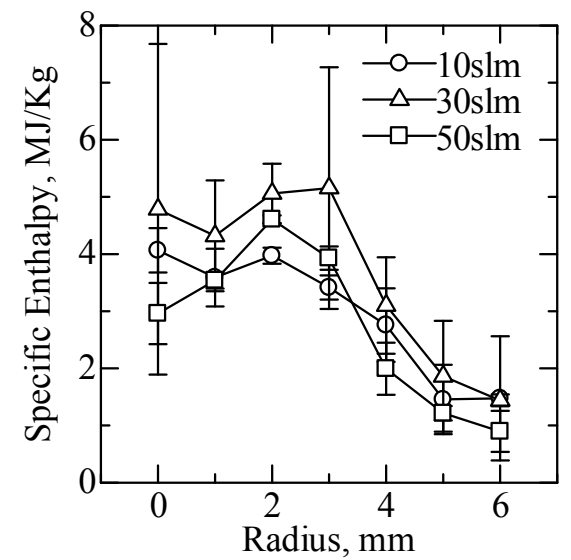

Fig. 11 Radial distribution of the specific enthalpy.

all species.

Using Eq. (5), $T_{0}$ distribution was deduced from measured $T$, $u$. Figure 11 shows the distributions of specific enthalpy. Figure shows that, there are no remarkable difference between three mass flow conditions, the $h_{0}$ was ranged from 3 to 5 $\mathrm{MJ} / \mathrm{kg}$ at the radial position $r<3 \mathrm{~mm}$, and it decreases gradually at the radial position $r>3 \mathrm{~mm}$. This is because, the velocity decreases along to the radial position, then the kinetic energy decreases.

\section{Conclusion}

Continuous wave laser driven wind tunnel was developed and high enthalpy flows were produced. As a result of operation tests, high enthalpy argon/oxygen flows were successfully generated with the high plenum pressure of 0.95 $\mathrm{MPa}$. And the maximum flux density is $2.2 \times 10^{21} / \mathrm{cm}^{2} \mathrm{~s}$ with 0.31 MPa plenum pressure.

Plume characteristics were diagnosed by laser absorption spectroscopy, and radial distribution of specific enthalpy was measured.

\section{References}

1) M. Matsui, T. Ikemoto, H. Takayanagi, K. Komurasaki, Y. Arakawa: Journal of Thermophysics and Heat Transfer, 2007 (In press)

2) G. Herdrich, M. Auweter-Kurtz: AIAA Paper 04-2503, Jun. 2004

3) T. Yamada, K. Fujita, S. Nonaka, N. Ishii: ISTS 2006-e-19.

4) K. Toyoda, K. Komurasaki, Y. Arakawa: Vacuum 65 (2002) 383.

5) W. Demtroeder: "Laser Spectroscopy," Basic Concepts and Instrumentation, 3rd ed., (Springer, Berlin, 2002) pp. 68-72.

6) M. Matsui: "Application of Laser Absorption Spectroscopy to High Enthalpy Flow Diagnostics,” Ph.D. Dissertation, Department of Advanced Energy, The University of Tokyo, Mar. 2005.

7) M. Matsui, O. Satoshi, K. Komurasaki, and Y. Arakawa: AIAA Paper 03-3903 (2003).

8) W Demtroder: Laser Spectroscopy, 2nd ed. (Springer Verlag, Berlin, 1996).

9) T. Inoue, T. Ijiri, S. Hosoda, K. Kojima, S. Uehara, K. Komurasaki, Y. Arakawa: Vacuum 73 (2004) 433.

10) M. Matsui, K. Komurasaki, G. Herdrich, and M. Auweter-Kurtz: AIAA Journal 43 (2005) 2060.

11) W. Demtroeder: "Laser Spectroscopy," Basic Concepts and Instrumentation, 3rd ed. (Springer, Berlin, 2002) pp. 68-72. 